\title{
Bath-Induced Correlations Enhance Thermometry Precision at Low Temperatures
}

\author{
Guim Planella, ${ }^{1,2,3}$ Marina F. B. Cenni®, ${ }^{1}$ Antonio Acín, ${ }^{1,4}$ and Mohammad Mehboudi $\circledast^{1,5,6, *}$ \\ ${ }^{1}$ ICFO-Institut de Ciencies Fotoniques, The Barcelona Institute of Science and Technology, 08860 Castelldefels (Barcelona), Spain \\ ${ }^{2}$ Facultat de Física, Universitat de Barcelona, 08028 Barcelona, Spain \\ ${ }^{3}$ Institute for Theoretical Physics, Utrecht University, 3584 CS Utrecht, Netherlands \\ ${ }^{4}$ ICREA-Institució Catalana de Recerca i Estudis Avançats, 08010 Barcelona, Spain \\ ${ }^{5}$ Max-Planck-Institut für Quantenoptik, D-85748 Garching, Germany \\ ${ }^{6}$ Département de Physique Appliquée, Université de Genève, 1211 Genève, Switzerland
}

(Received 3 June 2021; accepted 22 December 2021; published 24 January 2022)

\begin{abstract}
We study the role of bath-induced correlations in temperature estimation of cold bosonic baths. Our protocol includes multiple probes, that are not interacting, nor are they initially correlated to each other. They interact with a bosonic sample and reach a nonthermal steady state, which is measured to estimate the temperature of the sample. It is well known that in the steady state such noninteracting probes may get correlated to each other and even entangled. Nonetheless, the impact of these correlations in metrology has not been deeply investigated yet. Here, we examine their role for thermometry of cold bosonic gases and show that, although being classical, bath-induced correlations can lead to significant enhancement of precision for thermometry. The improvement is especially important at low temperatures, where attaining high precision thermometry is particularly demanding. The proposed thermometry scheme does not require any precise dynamical control of the probes and tuning the parameters and is robust to noise in initial preparation, as it is built upon the steady state generated by the natural dissipative dynamics of the system. Therefore, our results put forward new possibilities in thermometry at low temperatures, of relevance, for instance, in cold gases and Bose-Einstein condensates.
\end{abstract}

DOI: 10.1103/PhysRevLett.128.040502

Introduction.-Achieving extremely low temperatures is a must for quantum simulation and computation in many platforms. In order to fully characterize any system that works for such tasks, aside from tunable parameters one has to estimate the nontunable ones as well. Although these parameters vary depending on the platform, temperature is common among almost all, because thermal states naturally appear in many physical systems. Even if that is not the case, the statistics of subsystems of a quantum system often behave as if the quantum system was at thermal equilibrium [1-3]. Therefore, thermometry is a major focus of much theoretical and experimental research carried out in quantum systems [4-23].

Since quantum systems, especially when made of many constituents, are fragile and costly to prepare, the usage of small systems as probes is an essential method for nondestructively measuring their parameters [24]. As such, individual quantum probes for thermometry have been studied in several scenarios $[9,25,26]$ and their usefulness was recently demonstrated

Published by the American Physical Society under the terms of the Creative Commons Attribution 4.0 International license. Further distribution of this work must maintain attribution to the author(s) and the published article's title, journal citation, and DOI. Open access publication funded by the Max Planck Society. experimentally in ultracold gases [27]. When the probe thermalizes with the sample, universal results can be obtained thanks to the Gibbs ensemble, that connects thermometry precision to the heat capacity $[6,8]$. At very low temperatures, however, quantum probes do not thermalize with the sample; they rather reach a nonthermal steady state (NTSS) [28], which is generally model dependent [26,32]. Thermometry at low temperatures is timely and challenging. It has been shown that for thermal equilibrium probes, the error diverges exponentially as $T \rightarrow 0[6,8]$. Thus many attempts have been dedicated to overcome this limitation. In particular, when the probes are not at thermal equilibrium, it was shown that instead of exponential one can achieve a polynomial divergence $[11,22,23,26]$; still the error can be very large. Our work is thus motivated by these challenges, specifically regarding experimentally relevant bosonic models that describe impurity based thermometry of ultracold gases.

Most of the major experimental thermometry protocols that address ultracold gases use the time-of-flight absorption technique, which can be very precise, but is often destructive [33-35]. Nonetheless, there are some experiments in which an impurity is used as a probe. This impurity can be made up of multiple atoms that simultaneously interact with the system. They have been extensively studied and experimentally realized in both bosonic and fermionic gases [25,36-52]. 
It is well known that a quantum bath or sample can create correlations, and sometimes entanglement, among subsystems of a probe that interact with it. This can be the case even if the probes are initially uncorrelated and/or if they do not interact directly with one another. We call this phenomenon bath-induced correlations. In the past few years, several theoretical works have reported bath-induced correlations or entanglement at NTSS of different platforms including bosonic and fermionic environments [53-58] and even realized them experimentally [59]. However, to our knowledge, the use of such correlations to estimate the bath parameters has not been studied.

The main goal of our work is to analyze the thermometry of bosonic systems when using multiparticle probes and investigate the impact of bath-induced correlations in precision thermometry. We show that although entanglement-quantified by negativity-is absent, yet bathinduced correlations help significantly improving the precision of thermometry and in some cases leading to 2 orders of magnitude increase in the quantum Fisher information. These results can be used to address and improve nondemolition thermometry of Bose-Einstein condensates (BECs) in the $\mathrm{nK}$ and sub-nK domain aligned with previous efforts in characterizing correlations in BECs [58].

The setup and the model.-We consider a sample (bath) of bosonic harmonic oscillators. It is in a thermal state, and our aim is to estimate its temperature $T$ by bringing it in contact with an external probe. After sufficiently long interaction among the probe and the sample, the probe relaxes to its NTSS. Measurements are carried out solely on the probe, hence realizing a nondemolition measurement on the sample. Figure 1 illustrates the scenarios that we address here: (a) The independent-bath scenario, where no correlations are created among different oscillators as they lie in separate parts of the bath. (More precisely, we define this scenario as the case where the interprobe distances are larger than the correlation length of the bath.) This is our reference scenario. Any situation in which one invokes the thermalization assumption can be analyzed within this framework. (b) A more realistic scenario, where all of the probes are embedded in the same bath. If we increase the distance among neighboring probes, we expect to revive (a). Otherwise, this scenario gives rise to correlations among different oscillators - see below. This implies that using (a) to describe realistic protocols can lead to significant miscalculation of the thermometry precision. Moreover, while the thermometry precision in the independent-bath scenario (a) is additive, the correlations that appear in the common bath scenario (b) can lead to superadditive precision for thermometry giving rise to a significant enhancement.

Let us introduce details of the model and sketch how to exactly solve for the probe's NTSS (more details are provided in the Supplemental Material [60]; see also Refs. [26,61-63]). The total Hamiltonian reads (a)
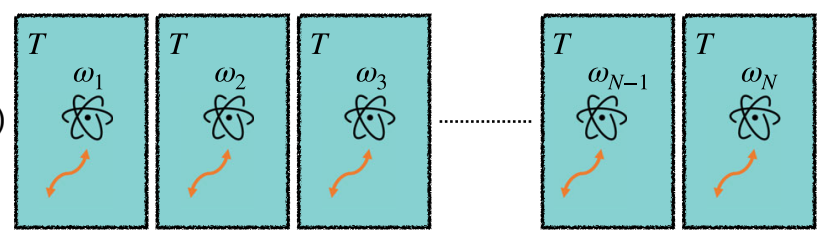

(b)

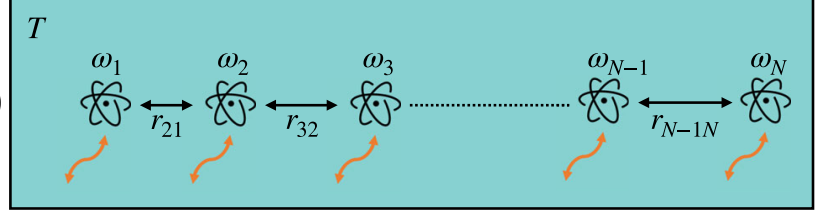

FIG. 1. Schematic of the thermometry protocols. (a) Independent baths: each thermometer is in contact with a separate bath, thus no correlations are built among them. Hence, the precision is additive. (b) Common bath-focus of this work: all thermometers are in contact with the same bath. The $i$ th and the $j$ th thermometers have a distance $r_{i j}$, they do not interact with each other nor do they share initial correlations, yet they get correlated thanks to their interactions with the common bath. Our results show that such correlations might lead to superadditive precision at low temperatures. The two scenarios are equivalent when the distance between the probes is very large or at high temperatures.

$$
H=H_{\mathrm{p}}+H_{\mathrm{B}}+H_{\mathrm{PB}},
$$

where the Hamiltonian of the 1D probe (see Ref. [57] for extension to higher dimensions) is

$$
H_{\mathrm{P}}=\sum_{i=1}^{N} \frac{p_{i}^{2}}{2 m_{i}}+\frac{1}{2} \sum_{i=1}^{N} m_{i} \omega_{i}^{2} x_{i}^{2}+\frac{1}{2} \sum_{i \neq j}^{N} g_{i j} x_{i} x_{j},
$$

with $x_{i}$ being the displacement from equilibrium position $r_{i}$ of the $i$ th probe oscillator and $p_{i}$ being the conjugate momentum. For now we allow for interoscillator interactions with couplings $g_{i j}$, but to study only bath-induced correlations we set $g_{i j}=0$ in simulations. The Hamiltonian of the bath reads

$$
H_{\mathrm{B}}=\sum_{k} \frac{q_{k}^{2}}{2 m_{k}}+\frac{1}{2} \sum_{k} m_{k} \omega_{k}^{2} y_{k}^{2},
$$

being $y_{k}$ and $q_{k}$ the position and momentum of the bath mode with the wave vector $k$. Generally, $H_{\mathrm{B}}$ could be an interacting model, however, if the interaction is quadratic, one can always bring it to the form (3) by finding its normal modes (e.g., see the 1D BECs studied in Refs. [9,64]). Finally, we consider a probe-bath interaction of the form

$$
H_{\mathrm{PB}}=\sum_{i=1}^{N} \sum_{k} G_{k} x_{i}\left(y_{k} \cos k r_{i}+\frac{q_{k}}{m_{k} \omega_{k}} \sin k r_{i}\right) \text {, }
$$

which is valid under the long wave approximation, where the wavelength of the bath excitations is much larger that 
the displacement of the oscillators from equilibrium, i.e., $\left|k x_{i}\right| \ll 1$ (see, e.g., Ref. [54]).

Equation (1) is quadratic, thus the dynamics is Gaussian and the NTSS will be Gaussian too. The NTSS does not depend on the initial state of the probe, it only depends on the total Hamiltonian, as well as the initial state of the bath, which we choose to be thermal at (unknown) temperature $T$. Since the NTSS is Gaussian, we only need the first and second order correlations - that is, the displacement vector and the covariance matrix, respectively - to fully describe it. If we define $R=\left(x_{1}, p_{1}, \ldots, x_{N}, p_{N}\right)^{T}$, then the displacement vector is a $2 N$ dimensional vector with elements $d_{i}=$ $\left\langle R_{i}\right\rangle$ while the covariance matrix is a $2 N \times 2 N$ symmetric matrix with elements $\Gamma_{i j}=\left\langle\left\{R_{i}, R_{j}\right\}\right\rangle / 2-\left\langle R_{i}\right\rangle\left\langle R_{j}\right\rangle$.

The conventional method of finding $d$ and $\Gamma$ starts by using the Heisenberg equations of motion-that for any observable $O$ reads $\dot{O}=i[H, O]$. Applying to all degrees of freedom gives

$$
\begin{gathered}
\dot{x}_{i}=\frac{p_{i}}{m_{i}}, \\
\dot{y}_{k}=\frac{q_{k}}{m_{k}}+\sum_{i=1}^{N} \frac{G_{k}}{m_{k} \omega_{k}} x_{i} \sin k r_{i}, \\
\dot{p}_{i}=-m_{i} \omega_{i}^{2} x_{i}-\sum_{j \neq i} g_{i j} x_{j} \\
-\sum_{k} G_{k}\left(y_{k} \cos k r_{i}+\frac{q_{k}}{m_{k} \omega_{k}} \sin k r_{i}\right), \\
\dot{q}_{k}=-m_{k} \omega_{k}^{2} y_{k}-\sum_{i=1}^{N} G_{k} x_{i} \cos k r_{i} .
\end{gathered}
$$

Solving these equations for the probe degrees of freedom gives the quantum Langevin equations of motion (see the Supplemental Material [60] and Refs. [62,65,66])

$$
m_{i} \ddot{x}_{i}+m_{i} \omega_{i}^{2} x_{i}+\sum_{j=1}^{N} g_{i j} x_{j}-\sum_{j=1}^{N} \chi_{i j} \star x_{j}=F_{i},
$$

where $\star$ stands for convolution. Here, the susceptibility matrix reads

$$
\chi_{i j}(t)=\sum_{k} \frac{G_{k}^{2}}{m_{k} \omega_{k}} \sin \left(\omega_{k} t+k r_{i j}\right) \Theta(t),
$$

where we define $r_{i j}:=r_{i}-r_{j}$, and the step function $\Theta(t)$ imposes causality. The susceptibility matrix is responsible for both memory effects-through the convolution-and correlations among the probes, even if $g_{i j}=0$. Finally, the vector of Brownian forces reads

$$
\begin{aligned}
F_{i}(t)= & -\sum_{k} G_{k}\left(y_{k}\left(t_{0}\right) \cos \left(\omega_{k}\left(t-t_{0}\right)+k r_{i}\right)\right. \\
& \left.+\frac{q_{k}\left(t_{0}\right)}{m_{k} \omega_{k}} \sin \left(\omega_{k}\left(t-t_{0}\right)+k r_{i}\right)\right) .
\end{aligned}
$$

The solution of (9) depends on the probe-bath interaction, and the particular spectral density describing it. The latter is a matrix with the elements

$$
J_{i j}(\omega)=\sum_{k} \frac{\pi G_{k}^{2}}{2 m_{k} \omega_{k}} \cos \left(k r_{i j}\right) \delta\left(\omega-\omega_{k}\right) .
$$

In what follows we consider a 1D bosonic bath with linear dispersion $k=\omega_{k} / c$, where $c$ denotes speed of sound. This corresponds to an Ohmic spectral density $[60,67,68]$

$$
J_{i j}(\omega)=\gamma^{2} \omega \frac{\Omega^{2}}{\omega^{2}+\Omega^{2}} \cos \frac{\omega\left|r_{i j}\right|}{c},
$$

with $\gamma$ the probe-Bath interaction strength and $\Omega$ the cutoff frequency. We exactly solve Eq. (9) and characterize the steady state by finding $d$ and $\Gamma$. First, we find that $d=0$. Second, if $\Gamma^{(\mathrm{a})}(T)$ and $\Gamma^{(\mathrm{b})}(T)$ are the temperature dependent covariance matrices in scenarios (a) and (b), respectively, we observe a major difference in their correlations: While in (a) we have an uncorrelated state with $\Gamma^{(\mathrm{a})}(T)=\bigoplus_{i} \sigma_{i}^{(\mathrm{a})}(T)-\sigma_{i}^{(\alpha)}(T)$ being the local covariance matrix of the $i$ th probe in scenario $\alpha \in\{\mathrm{a}, \mathrm{b}\}$-this is not the case for (b), in which interoscillator correlations appear. These correlations disappear at large distance, and/or at high temperatures. Nonetheless, at low temperature regimes, and small distances they can be significantsee Ref. [60] for details — and are responsible for enhanced thermometry precision. Interestingly, we find out that entanglement negativity, for arbitrary bipartitions, is zero. Nonetheless, classical correlations are present in the common bath scenario and we show below that this major difference in the correlation structure significantly enhances thermometry precision.

Metrology in Gaussian quantum systems.-We are dealing with parameter estimation in bosonic Gaussian quantum systems. Let $\Gamma(\lambda)$ be the covariance matrix of a Gaussian quantum system. Here, $\lambda$ is to be estimated, which can be temperature or any other parameter. We drop the parameter dependence of the covariance matrix to have a lighter notation, and restrict ourselves to scenarios with $d=0$, as is the case in our problem.

For a given measurement, with the measurement operator set $\left\{\Pi^{s}(o)\right\}$-where $s$ labels the specific measurement, and $o$ denotes different outcomes which can be continuous or discrete- the error on estimation of $\lambda$ is bounded from below by [69] 


$$
\delta \lambda(s) \geq \frac{1}{\nu \sqrt{\mathcal{F}^{\mathrm{cl}}(\lambda, s)}} \geq \frac{1}{\nu \sqrt{\mathcal{F}^{Q}(\lambda)}}=: \delta \lambda_{\min }
$$

where $\nu$ is the number of measurements, and $\mathcal{F}^{\mathrm{cl}}(\lambda, s)$ is the classical Fisher information (CFI) associated with the performed measurement defined as

$$
\mathcal{F}^{\mathrm{cl}}(\lambda, s):=\left\langle\left[\partial_{\lambda} \log p(o \mid s, \lambda)\right]^{2}\right\rangle_{p(o \mid s, \lambda)} .
$$

Here, $p(o \mid s, \lambda)$ is the conditional probability of observing $o$ given the parameter has the value $\lambda$ and the measurement $s$ is performed. The quantity $\mathcal{F}^{Q}(\lambda):=\max _{s} \mathcal{F}^{\mathrm{cl}}(\lambda, s)$ is the quantum Fisher information (QFI) that is obtained by maximizing the CFI over all measurements and thus is independent of $s$. The first inequality in Eq. (14) is called the Cramer-Rao bound, which can be saturated by suitable postprocessing of the outcomes. Thus, CFI can be a precision quantifier for a given measurement. The second inequality is the quantum Cramer-Rao bound that sets a fundamental lower bound on the error, regardless of the measurement. Importantly, this bound can be also saturated, by definition of the QFI.

Finding the optimal measurement and the QFI is challenging and requires different approaches depending on the platform, the underlying dynamics, and the specific parameter to be estimated. Nonetheless, for Gaussian systems, one can routinely find them [60,70-74]. In Ref. [60] we show that the optimal measurement is given by a linear combination of second order quadratures, which is highly nonlocal and experimentally demanding. Thus we also examine practically feasible alternatives, namely, local position and momentum measurements defined as $X=$ $\bigotimes_{i=1}^{N} x_{i}$ and $P=\bigotimes_{i=1}^{N} p_{i}$, respectively. These belong to the family of Gaussian measurements-i.e., when measuring Gaussian systems their outcomes are Gaussian distributed - for which the CFI is straightforwardly calculable $[60,70,74]$. Despite being suboptimal measurements, they can benefit from the enhancement, which puts forward an experimentally realizable scheme to significantly enhancing thermometry of bosonic systems.

Enhanced thermometry with bath-induced correlations. - We study the single shot $(\nu=1)$ relative error $\delta T_{\min } / T$ for a variety of parameters. Figure 2 illustrates $\delta T_{\min } / T$ versus temperature for various probe-bath couplings. For both scenarios (a) and (b), we observe that at high temperatures $\delta T_{\min } / T$ increases by increasing the coupling, whereas at low temperatures the opposite is observed. This unifies the findings of Refs. [9] and [26], and extends them from the single to the multiprobe scenario. Furthermore, Fig. 2 shows that at lower temperatures and for a fixed coupling, the scenario (b) always outperforms (a). To see this more clearly, we fix the coupling, and depict the normalized error $\sqrt{N} \delta T_{\min } / T$ in Fig. 3. At small temperatures, we observe that in scenario (b) the normalized error reduces by increasing the number

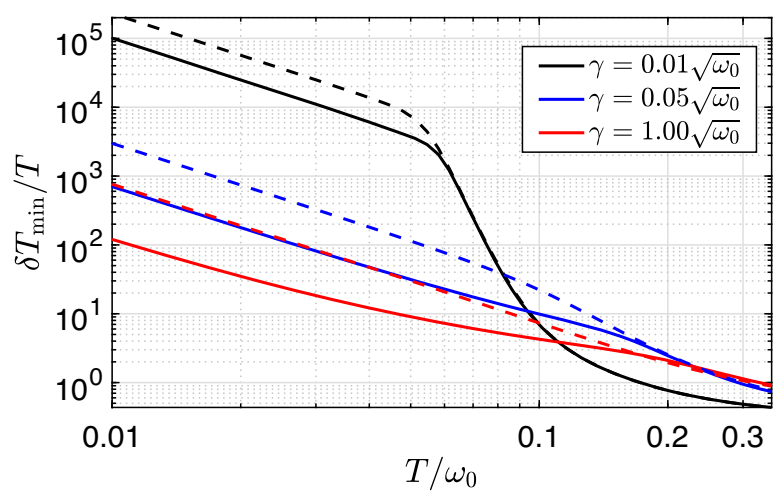

FIG. 2. Minimum relative error $\left[\delta T_{\min } / T\right.$ from r.h.s of Eq. (14)] against temperature for different system-bath couplings $\gamma$ (quantitative description in the Supplemental Material [60]) for a fixed number of oscillators. The solid curves represent the common bath scenario (b), whereas the dashed lines are obtained considering independent baths scenario (a). We see that at low temperatures by increasing $\gamma$ the error reduces, whereas at higher temperatures the opposite holds (not shown). Moreover, embedding the oscillators at the same bath can significantly decrease the relative error. Here, we set the parameters to $\omega_{0}=1$ in arbitrary units, $\quad \omega_{i}=\omega_{0} \forall i, \quad r_{21} / c \omega_{0}=0.1, \quad \Omega=100 \omega_{0}, \quad g_{i j}=0$, and $N=10$.

of probes and thus significantly outperforming scenario (a). By increasing $T$, the enhancement is progressively lost and even the scenario (a) might become slightly better. At even higher temperatures, the probes thermalize with the bath, and the two scenarios become equivalent as expected.

In Fig. 4 we fix the temperature in the low-temperature limit, and depict the QFI versus the number of oscillators.

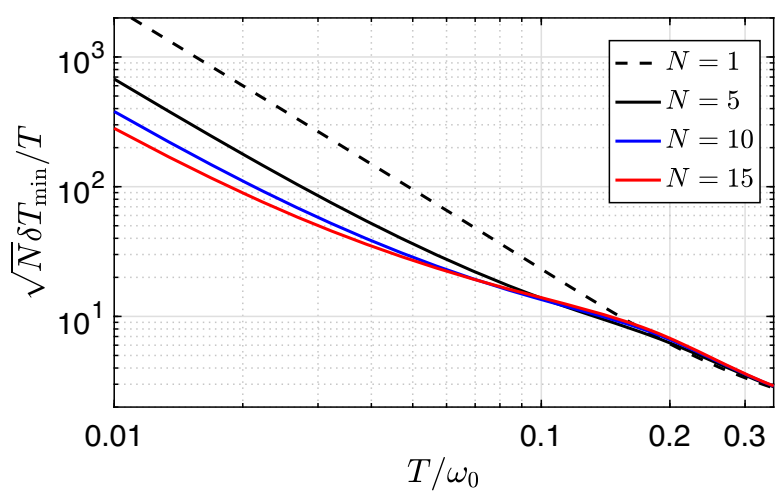

FIG. 3. Minimum relative error normalized by the number of oscillators (that is $\sqrt{N} \delta T_{\min } / T$ ), as a function of temperature, in the independent baths scenario (a) for arbitrary $N$ (dashed black) and in the common bath scenario (b): for different number of oscillators $N=1$ (also dashed black), $N=5$ (solid black), $N=10$ (solid blue), and $N=15$ (solid red). Unlike the scenario (a) which is additive, the scenario (b) is superadditive, as the normalized error reduces by increasing $N$. The parameters are set to $\omega_{0}=1$ in arbitrary units, and $\omega_{i}=\omega_{0} \forall i, r_{21} / c \omega_{0}=0.1$, $\Omega=100 \omega_{0}, g_{i j}=0$, and $\gamma=\sqrt{\omega_{0}}$. 


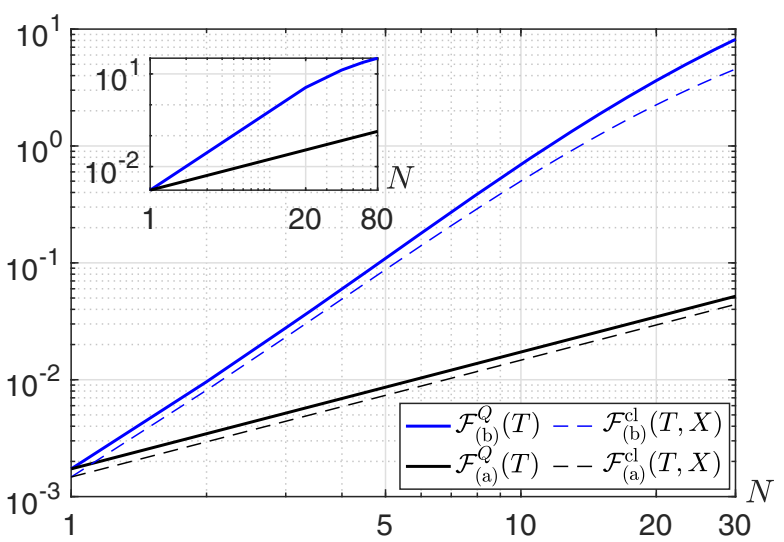

FIG. 4. Log-log plot of the quantum Fisher information vs $N$ in solid blue for scenario (b) and solid black for scenario (a). Here, we tune the setting to the low temperature limit with $T_{0}=0.01 \omega_{0}$. Scenario (b) behaves superlinearly; our data fitting shows that $\mathcal{F}\left(T_{0}\right) \propto N^{2.5}$ in the range of $1<N<30$, although the slope is decreasing as we see for $N \approx 80$ we have $\mathcal{F}\left(T_{0}\right) \propto$ $N^{1.1}$ (Inset). Nonetheless, for $N=30$ we already see that the QFI in scenario (b) is 2 orders of magnitude bigger than (a) — which shows a linear behavior as suggested by the additivity of the QFI for a tensor product state. The CFI for measuring $X=\otimes_{i=1}^{N} x_{i}$ is also depicted in dashed black for (a) and dashed blue for (b). Albeit suboptimal, these local measurements show a similar behavior to the optimal ones in both scenarios. Specifically, for scenario (b) they are superlinear with $N$. The rest of the parameters are set to $\omega_{0}=1, \quad \omega_{i}=\omega_{0} \forall i, \quad \gamma=\sqrt{\omega_{0}}$, $\Omega=100 \omega_{0}, g_{i j}=0$, and $r_{21} / c \omega_{0}=0.1$.

The slope of the QFI in the common bath scenario (b) is clearly larger than scenario (a). For $1<N<30$, on average, $\mathcal{F}^{Q}(T) \propto N^{2.5}$. However, as seen from the inset, the slope decreases for larger $N$ and it reaches $N^{1.1}$ for $N \approx 80$. Nonetheless, for $N \geq 30$ we see at least 2 orders of magnitude growth in the QFI due to the bath-induced correlations. It is worth mentioning that in the literature there are few works that study the role of correlations for thermometry in dynamical scenarios $[15,16]$ that might need high degrees of control; but to our knowledge this is the first time bath-induced correlations at NTSS are exploited for thermometry - and even other metrological tasks. What is more interesting is that the entanglement, as characterized by logarithmic negativity, is zero among any possible bipartition, hence, the enhancement is not due to quantum correlations.

Generally, the optimal measurement can be highly nonlocal. This is indeed the case for our common bath scenario (b); see the Supplemental Material [60] where we also cite Refs. [75-77]. For experimental purposes we also find simple local measurements that exploit the bathinduced correlations for enhanced thermometry at low temperatures. Particularly, we examine the CFI of local position and momentum measurements (only position measurement is shown here.) The result is depicted in Fig. 4. Although being suboptimal, the precision is superadditive and is better than the QFI of scenario (a) already for $N \geq 2$, demonstrating the advantage of exploiting bath-induced correlations even with local Gaussian measurements.

Discussion.-We showed that bath-induced correlations present in the steady state of thermometers can play a prominent role in thermometry of bosonic baths at low temperatures, which is where precision thermometry is known to be most demanding. Although these correlations are not of a quantum nature, they can increase the thermometry precision up to 2 orders of magnitude, for both optimal and experimentally feasible measurements. In our scheme, neither preparation of highly entangled states, nor dynamical control is required-compared to alternative dynamical based proposals $[15,16,18,25]$ — which makes them very appealing. Unfortunately, unlike typical phase estimation problems, the steady state depends on the temperature nontrivially. This makes it challenging to pinpoint the intrinsic properties of the bath and the steady state that intensify the impact of correlations in thermometry precision. Therefore, an interesting future direction is to consider properties of covariance matrices and their temperature dependence (regardless of any physical model in the background) that leads to such improvements. It would be also interesting to investigate other spectral densities-corresponding to physically relevant models, e.g., with alternative spacial dimension and trapping potential-and see whether or how they affect the impact of the common bath on thermometry precision. Bathinduced correlations for thermometry might be examined in several experimental setups e.g., Refs. [13,37,42,78-80] and possibly explored in fermionic platforms [36,81-83].

Constructive discussions with M. A. Garcia-March, C. Charalambous, S. Iblisdir, S. Hérnandez-Santana, D. Alonso, and L.A. Correa are appreciated. This work was financially supported by the Government of Spain (ConTrAct FIS2017-83709-R, FIS2020-TRANQI and Severo Ochoa CEX2019-000910-S), the ERC AdG CERQUTE, the AXA Chair in Quantum Information Science, Fundacio Cellex, Fundacio Mir-Puig, Generalitat de Catalunya (CERCA, AGAUR SGR 1381 and QuantumCAT) and the Swiss National Science Foundations (NCCR SwissMAP).

*mohammad.mehboudi@unige.ch

[1] C. Gogolin and J. Eisert, Equilibration, thermalisation, and the emergence of statistical mechanics in closed quantum systems, Rep. Prog. Phys. 79, 056001 (2016).

[2] S. Popescu, A. J. Short, and A. Winter, Entanglement and the foundations of statistical mechanics, Nat. Phys. 2, 754 (2006).

[3] S. Goldstein, J. L. Lebowitz, R. Tumulka, and N. Zanghì, Canonical typicality, Phys. Rev. Lett. 96, 050403 (2006). 
[4] M. Mehboudi, A. Sanpera, and L. A. Correa, Thermometry in the quantum regime: Recent theoretical progress, J. Phys. A 52, 303001 (2019).

[5] A. De Pasquale and T. M. Stace, Quantum thermometry, Thermodynamics in the Quantum Regime (Springer, New York, 2018), pp. 503-527.

[6] L. A. Correa, M. Mehboudi, G. Adesso, and A. Sanpera, Individual Quantum Probes for Optimal Thermometry, Phys. Rev. Lett. 114, 220405 (2015).

[7] A. De Pasquale, D. Rossini, R. Fazio, and V. Giovannetti, Local quantum thermal susceptibility, Nat. Commun. 7, 12782 (2016).

[8] M. G. A. Paris, Achieving the Landau bound to precision of quantum thermometry in systems with vanishing gap, J. Phys. A 49, 03LT02 (2015).

[9] M. Mehboudi, A. Lampo, C. Charalambous, L. A. Correa, M. Á. García-March, and M. Lewenstein, Using Polarons for Sub-Nk Quantum Nondemolition Thermometry in a Bose-Einstein Condensate, Phys. Rev. Lett. 122, 030403 (2019).

[10] S. Razavian, C. Benedetti, M. Bina, Y. Akbari-Kourbolagh, and M. G. A. Paris, Quantum thermometry by single-qubit dephasing, Eur. Phys. J. Plus 134, 284 (2019).

[11] K. V. Hovhannisyan and L. A. Correa, Measuring the temperature of cold many-body quantum systems, Phys. Rev. B 98, 045101 (2018).

[12] M. Mehboudi, M. Moreno-Cardoner, G. De Chiara, and A. Sanpera, Thermometry precision in strongly correlated ultracold lattice gases, New J. Phys. 17, 055020 (2015).

[13] S. S. Mirkhalaf, D. B. Orenes, M. W. Mitchell, and E. Witkowska, Criticality-enhanced quantum sensing in ferromagnetic Bose-Einstein condensates: Role of readout measurement and detection noise, Phys. Rev. A 103, 023317 (2021).

[14] P. P. Hofer, J. B. Brask, M. Perarnau-Llobet, and N. Brunner, Quantum Thermal Machine as a Thermometer, Phys. Rev. Lett. 119, 090603 (2017).

[15] S. Seah, S. Nimmrichter, D. Grimmer, J. P. Santos, V. Scarani, and G. T. Landi, Collisional Quantum Thermometry, Phys. Rev. Lett. 123, 180602 (2019).

[16] G. A. Paz-Silva, L. M. Norris, and L. Viola, Multiqubit spectroscopy of Gaussian quantum noise, Phys. Rev. A 95, 022121 (2017).

[17] S. Campbell, M. Mehboudi, G. De Chiara, and M. Paternostro, Global and local thermometry schemes in coupled quantum systems, New J. Phys. 19, 103003 (2017).

[18] M. T. Mitchison, T. Fogarty, G. Guarnieri, S. Campbell, T. Busch, and J. Goold, In Situ Thermometry of a Cold Fermi Gas via Dephasing Impurities, Phys. Rev. Lett. 125, 080402 (2020).

[19] W.-K. Mok, K. Bharti, L.-C. Kwek, and A. Bayat, Optimal probes for global quantum thermometry, Commun. Phys. 4, 1 (2021).

[20] C. L. Latune, I. Sinayskiy, and F. Petruccione, Collective heat capacity for quantum thermometry and quantum engine enhancements, New J. Phys. 22, 083049 (2020).

[21] J. Rubio, J. Anders, and L. A. Correa, Global Quantum Thermometry, Phys. Rev. Lett. 127, 190402 (2021).
[22] P. P. Potts, J. B. Brask, and N. Brunner, Fundamental limits on low-temperature quantum thermometry with finite resolution, Quantum 3, 161 (2019).

[23] M. R. Jørgensen, P. P. Potts, M. G. A. Paris, and J. B. Brask, Tight bound on finite-resolution quantum thermometry at low temperatures, Phys. Rev. Research 2, 033394 (2020).

[24] V. Giovannetti, S. Lloyd, and L. Maccone, Advances in quantum metrology, Nat. Photonics 5, 222 (2011).

[25] V. Mukherjee, A. Zwick, A. Ghosh, X. Chen, and G. Kurizki, Enhanced precision bound of low-temperature quantum thermometry via dynamical control, Commun. Phys. 2, 1 (2019).

[26] L. A. Correa, M. Perarnau-Llobet, K. V. Hovhannisyan, S. Hernández-Santana, M. Mehboudi, and A. Sanpera, Enhancement of low-temperature thermometry by strong coupling, Phys. Rev. A 96, 062103 (2017).

[27] Q. Bouton, J. Nettersheim, D. Adam, F. Schmidt, D. Mayer, T. Lausch, E. Tiemann, and A. Widera, Single-Atom Quantum Probes for Ultracold Gases Boosted by Nonequilibrium Spin Dynamics, Phys. Rev. X 10, 011018 (2020).

[28] Here, we refer to the reduced state of the probe as nonthermal steady state (NTSS) to emphasize that it cannot be written as a Gibbs state with the Hamiltonian $H_{p}$. To avoid confusion, we do not use the terminology nonequilibrium steady state (NESS), because unlike the latter, here the total system Bath might be described by a Gibbs state-roughly speaking, the set of NTSSs contains NESSs. See, e.g., Refs. [29-31] for further details.

[29] W. Aschbacher, V. Jakšić, Y. Pautrat, and C.-A. Pillet, Topics in non-equilibrium quantum statistical mechanics, Open Quantum Systems III (Springer, New York, 2006), pp. 1-66.

[30] T. Monnai, S. Morodome, and K. Yuasa, Relaxation to Gaussian generalized Gibbs ensembles in quadratic bosonic systems in the thermodynamic limit, Phys. Rev. E 100, 022105 (2019).

[31] V. Bach, J. Fröhlich, and I. M. Sigal, Return to equilibrium, J. Math. Phys. (N.Y.) 41, 3985 (2000).

[32] H.-P. Breuer and F. Petruccione, The Theory of Open Quantum Systems (Oxford University Press, New York, 2002).

[33] A. E. Leanhardt, T. A. Pasquini, M. Saba, A. Schirotzek, Y. Shin, D. Kielpinski, D. E. Pritchard, and W. Ketterle, Cooling Bose-Einstein condensates below 500 picokelvin, Science 301, 1513 (2003).

[34] R. Gati, B. Hemmerling, J. Fölling, M. Albiez, and M. K. Oberthaler, Noise Thermometry with Two Weakly Coupled Bose-Einstein Condensates, Phys. Rev. Lett. 96, 130404 (2006).

[35] R. Gati, J. Esteve, B. Hemmerling, T. B. Ottenstein, J. Appmeier, A. Weller, and M. K. Oberthaler, A primary noise thermometer for ultracold bose gases, New J. Phys. 8, 189 (2006).

[36] F. M. Spiegelhalder, A. Trenkwalder, D. Naik, G. Hendl, F. Schreck, and R. Grimm, Collisional Stability of ${ }^{40} \mathbf{K}$ Immersed in a Strongly Interacting Fermi gas of ${ }^{6} \mathrm{Li}$, Phys. Rev. Lett. 103, 223203 (2009).

[37] R. Olf, F. Fang, G. E. Marti, A. MacRae, and D. M. Stamper-Kurn, Thermometry and cooling of a Bose gas 
to 0.02 times the condensation temperature, Nat. Phys. 11, 720 (2015).

[38] P. Massignan, M. Zaccanti, and G. M. Bruun, Polarons, dressed molecules and itinerant ferromagnetism in ultracold Fermi gases, Rep. Prog. Phys. 77, 034401 (2014).

[39] N. B. Jørgensen, L. Wacker, K. T. Skalmstang, M. M. Parish, J. Levinsen, R. S. Christensen, G. M. Bruun, and J. J. Arlt, Observation of Attractive and Repulsive Polarons in a Bose-Einstein Condensate, Phys. Rev. Lett. 117, 055302 (2016).

[40] R. Côté, V. Kharchenko, and M. D. Lukin, Mesoscopic Molecular Ions in Bose-Einstein Condensates, Phys. Rev. Lett. 89, 093001 (2002).

[41] T. Rentrop, A. Trautmann, F. A. Olivares, F. Jendrzejewski, A. Komnik, and M. K. Oberthaler, Observation of the Phononic Lamb Shift with a Synthetic Vacuum, Phys. Rev. X 6, 041041 (2016).

[42] J. Catani, G. Lamporesi, D. Naik, M. Gring, M. Inguscio, F. Minardi, A. Kantian, and T. Giamarchi, Quantum dynamics of impurities in a one-dimensional Bose gas, Phys. Rev. A 85, 023623 (2012).

[43] R. Schmidt, T. Enss, V. Pietilä, and E. Demler, Fermi polarons in two dimensions, Phys. Rev. A 85, 021602(R) (2012).

[44] M.-G. Hu, M. J. Van de Graaff, D. Kedar, J. P. Corson, E. A. Cornell, and D.S. Jin, Bose Polarons in the Strongly Interacting Regime, Phys. Rev. Lett. 117, 055301 (2016).

[45] P. Massignan, C. J. Pethick, and H. Smith, Static properties of positive ions in atomic Bose-Einstein condensates, Phys. Rev. A 71, 023606 (2005).

[46] F. M. Cucchietti and E. Timmermans, Strong-Coupling Polarons in Dilute Gas Bose-Einstein Condensates, Phys. Rev. Lett. 96, 210401 (2006).

[47] N. Spethmann, F. Kindermann, S. John, C. Weber, D. Meschede, and A. Widera, Dynamics of Single Neutral Impurity Atoms Immersed in an Ultracold Gas, Phys. Rev. Lett. 109, 235301 (2012).

[48] S. P. Rath and R. Schmidt, Field-theoretical study of the Bose polaron, Phys. Rev. A 88, 053632 (2013).

[49] D. Benjamin and E. Demler, Variational polaron method for Bose-Bose mixtures, Phys. Rev. A 89, 033615 (2014).

[50] R. S. Christensen, J. Levinsen, and G. M. Bruun, Quasiparticle Properties of a Mobile Impurity in a Bose-Einstein Condensate, Phys. Rev. Lett. 115, 160401 (2015).

[51] J. Levinsen, M. M. Parish, and G. M. Bruun, Impurity in a Bose-Einstein Condensate and the Efimov Effect, Phys. Rev. Lett. 115, 125302 (2015).

[52] Y. E. Shchadilova, R. Schmidt, F. Grusdt, and E. Demler, Quantum Dynamics of Ultracold Bose Polarons, Phys. Rev. Lett. 117, 113002 (2016).

[53] A. Wolf, G. De Chiara, E. Kajari, E. Lutz, and G. Morigi, Entangling two distant oscillators with a quantum reservoir, Europhys. Lett. 95, 60008 (2011).

[54] A. A. Valido, D. Alonso, and S. Kohler, Gaussian entanglement induced by an extended thermal environment, Phys. Rev. A 88, 042303 (2013).
[55] B. Kraus, H. P. Büchler, S. Diehl, A. Kantian, A. Micheli, and P. Zoller, Preparation of entangled states by quantum Markov processes, Phys. Rev. A 78, 042307 (2008).

[56] M. Ludwig, K. Hammerer, and F. Marquardt, Entanglement of mechanical oscillators coupled to a nonequilibrium environment, Phys. Rev. A 82, 012333 (2010).

[57] A. A. Valido, A. Ruiz, and D. Alonso, Quantum correlations and energy currents across three dissipative oscillators, Phys. Rev. E 91, 062123 (2015).

[58] C. Charalambous, M. A. Garcia-March, A. Lampo, M. Mehboudi, and M. Lewenstein, Two distinguishable impurities in BEC: squeezing and entanglement of two Bose polarons, SciPost Phys. 6, 10 (2019).

[59] H. Krauter, C. A. Muschik, K. Jensen, W. Wasilewski, J. M. Petersen, J. I. Cirac, and E. S. Polzik, Entanglement generated by Dissipation and Steady State Entanglement of Two Macroscopic Objects, Phys. Rev. Lett. 107, 080503 (2011).

[60] See Supplemental Material at http://link.aps.org/ supplemental/10.1103/PhysRevLett.128.040502 for (A) Derivation of the Langevin equations, the fluctuationdissipation theorem, the steady states. The temperature and distance behaviour of the correlations are also presented. (B) Details of parameter estimation in quantum Gaussian systems.

[61] U. Weiss, Quantum Dissipative Systems (World Scientific, Singapore, 2012), Vol. 13.

[62] H. P. Breuer and F. Petruccione, The Theory of Open Quantum Systems (Oxford University Press, Oxford, 2007).

[63] C. Weedbrook, S. Pirandola, R. García-Patrón, N. J. Cerf, T. C. Ralph, J. H. Shapiro, and S. Lloyd, Gaussian quantum information, Rev. Mod. Phys. 84, 621 (2012).

[64] A. Lampo, S. H. Lim, M. Á. García-March, and M. Lewenstein, Bose polaron as an instance of quantum Brownian motion, Quantum 1, 30 (2017).

[65] D. S. Lemons and A. Gythiel, Paul Langevin's 1908 paper "On the Theory of Brownian Motion" ["Sur la théorie du mouvement brownien," C. R. Acad. Sci. (Paris) 146, 530533 (1908)], Am. J. Phys. 65, 1079 (1997).

[66] P. Langevin, Sur la theorie de mouvement brownien, C. R. Acad. Sci. (Paris) 146, 530 (1908).

[67] J. O. González, L. A. Correa, G. Nocerino, J. P. Palao, D. Alonso, and G. Adesso, Testing the validity of the 'local' and 'global'gkls master equations on an exactly solvable model, Open Syst. Inf. Dyn. 24, 1740010 (2017).

[68] U. Kleinekathöfer, Non-Markovian theories based on a decomposition of the spectral density, J. Chem. Phys. 121, 2505 (2004).

[69] M. G. A. Paris, Quantum estimation for quantum technology, Int. J. Quantum. Inform. 07, 125 (2009).

[70] A. Monras, Phase space formalism for quantum estimation of Gaussian states, arXiv:1303.3682.

[71] Z. Jiang, Quantum Fisher information for states in exponential form, Phys. Rev. A 89, 032128 (2014).

[72] R. Nichols, P. Liuzzo-Scorpo, P. A. Knott, and G. Adesso, Multiparameter Gaussian quantum metrology, Phys. Rev. A 98, 012114 (2018).

[73] L. Malagò and G. Pistone, Information geometry of the gaussian distribution in view of stochastic optimization, 
Proceedings of the 2015 ACM Conference on Foundations of Genetic Algorithms XIII (Association for Computing Machinery, New York, 2015), pp. 150-162.

[74] M. F. B. Cenni, L. Lami, A. Acin, and M. Mehboudi, Thermometry of Gaussian quantum systems using Gaussian measurements, arXiv:2110.02098.

[75] P. Giorda and M. G. A. Paris, Gaussian Quantum Discord, Phys. Rev. Lett. 105, 020503 (2010).

[76] G. Adesso and D. Girolami, Gaussian geometric discord, Int. J. Quantum. Inform. 09, 1773 (2011).

[77] S. Rahimi-Keshari, C. M. Caves, and T. C. Ralph, Measurement-based method for verifying quantum discord, Phys. Rev. A 87, 012119 (2013).

[78] A. P. Chikkatur, A. Görlitz, D. M. Stamper-Kurn, S. Inouye, S. Gupta, and W. Ketterle, Suppression and Enhancement of Impurity Scattering in a Bose-Einstein Condensate, Phys. Rev. Lett. 85, 483 (2000).
[79] R. Scelle, T. Rentrop, A. Trautmann, T. Schuster, and M. K. Oberthaler, Motional Coherence of Fermions Immersed in a Bose Gas, Phys. Rev. Lett. 111, 070401 (2013).

[80] A. Shashi, F. Grusdt, D. A. Abanin, and E. Demler, Radiofrequency spectroscopy of polarons in ultracold Bose gases, Phys. Rev. A 89, 053617 (2014).

[81] A. Schirotzek, C.-H. Wu, A. Sommer, and M. W. Zwierlein, Observation of Fermi Polarons in a Tunable Fermi Liquid of Ultracold Atoms, Phys. Rev. Lett. 102, 230402 (2009).

[82] C. Kohstall, M. Zaccanti, M. Jag, A. Trenkwalder, P. Massignan, G. M. Bruun, F. Schreck, and R. Grimm, Metastability and coherence of repulsive polarons in a strongly interacting Fermi mixture, Nature (London) 485, 615 (2012).

[83] M. Koschorreck, D. Pertot, E. Vogt, B. Fröhlich, M. Feld, and M. Köhl, Attractive and repulsive Fermi polarons in two dimensions, Nature (London) 485, 619 (2012). 